Nur als Zusammenfassung publiziert wurden folgende Arbeiten:

DECAE, A. E.: The trapdoor-burrow: the success of a defense system LANG, A.: Silk of the nuptial gift of the spider Pisaura mirabilis

MAURER, R.: Zur Gattung Cybaeus im Alpenraum (Araneae: Agelenidae, Cybaeinae) ZWÖRNER-WEICKMANN, D. \& S. MOOSMANN: Neue Erfahrungen mit Giftspinnen ZWÖRNER-WEICKMANN, D., S. MOOSMANN \& M. FORMELLA: Springspinnen: Interessatte Beobachtungen bei der Haltung und Zucht von Salticiden

Der Redaktion ist eine ansprechende Gestaltung sowohl des Bandes als auch der einzelnen Beiträge gelungen. Pierre-Alain FÜRST und Gilles MULHAUSER gebührt Dank für diese Leistung! Leider wird der Gesamteindruck durch einige Druckfehler etwas geschmälert. Sie sind offensichtlich in den meisten Fällen auf Probleme der computergestützten Textbearbeitung zurückzuführen.

Peter BLISS

\title{
Aufruf zur Mitarbeit
}

Wer einige von mir neu erstellte Bestimmungs-Tabellen mitteleuropäischer Spinnen (Gattungen, Arten) vor ihrer Publikation auf Brauchbarkeit/Fehler überprüfen möchte, melde sich bitte bei:

Jörg WUNDERLICH, Hindenburgstr. 94, D-75334 Straubenhardt Tel. 07082/3411.

\section{Aufruf zur Mitarbeit}

Gesucht werden Tiere der Art Pardosa (Wadicosa) venatrix. Wer kann mir bitte ein Paar dieser im Mittelmeergebiet vorkommenden Wolfspinnenart überlassen oder leihweise zusenden?

Dr. Hozumi TANAKA, Sonoda Gakuen Womens's College, 29-1, 7 chome Minamitsukaguchi-cho, Amagasaki-shi, Hyogo 661, JAPAN 